\title{
Proximinality in Banach space valued Musielak-Orlicz spaces
}

Jingshi $\mathrm{Xu}^{*}$

\section{"Correspondence:}

jingshixu@126.com

Department of Mathematics,

Hainan Normal University, Haikou,

571158, China

\section{Springer}

\begin{abstract}
Let $(A, \mathcal{A}, \mu)$ be a $\sigma$-finite complete measure space and let $Y$ be a subspace of a Banach space $X$. Let $\varphi$ be a generalized $\Phi$-function on $(A, \mathcal{A}, \mu)$. Denote by $L^{\varphi}(A, Y)$ and $L^{\varphi}(A, X)$ the Musielak-Orlicz spaces whose functions take values in $Y$ and $X$, respectively. Firstly, let $f \in L^{\varphi}(A, X)$, we characterize the distance of $f$ from $L^{\varphi}(A, Y)$. Then, if $Y$ is weakly $\mathcal{K}$-analytic and proximinal in $X$, we show that $L^{\varphi}(A, Y)$ is proximinal in $L^{\varphi}(A, X)$. Finally, we give the connection between the proximinality of $L^{\varphi}(A, Y)$ in $L^{\varphi}(A, X)$ and the proximinality of $L^{1}(A, Y)$ in $L^{1}(A, X)$.
\end{abstract}

Keywords: proximinality; Musielak-Orlicz space; best approximation; weakly $\mathcal{K}$-analytic

\section{Introduction}

It is well known that Musielak-Orlicz spaces include many spaces as special spaces, such as Lebesgue spaces, weighted Lebesgue spaces, variable Lebesgue spaces and Orlicz spaces; see [1]. Especially, in recent decades, variable exponent function spaces, such as Lebesgue, Sobolev, Besov, Triebel-Lizorkin, Hardy, Morrey, and Herz spaces with variable exponents, have attracted much attention; see [2-16] and references therein. Cheng and the author discussed geometric properties of Banach space valued Bochner-Lebesgue and Bochner-Sobolev spaces with a variable exponent in [17]. Very recently, Musielak-OrliczHardy spaces have been systemically developed; see, for example, [18-22]. These spaces have many applications in various fields such as PDE, electrorheological fluids, and image restoration; see [6, 23-25].

In recent years, proximinality in Banach space valued Bochner-Lebesgue spaces with constant exponent have been extensively studied; see [26-33]. Proximinality in Banach space valued Bochner-Lebesgue spaces with variable exponent was discussed by the author in [34]. In fact, we generalized those results in [29, 31] to Banach space valued Bochner-Lebesgue spaces with a variable exponent. Khandaqji, Khalil and Hussein considered proximinality in Orlicz-Bochner function spaces on the unit interval in [35], and Al-Minawi and Ayesh consider the same problem on finite measures in [36]. The best simultaneous approximation in Banach space valued Orlicz spaces was discussed in [37, 38]. Micherda discussed proximinality of subspaces of vector-valued Musielak-Orlicz spaces via modular in [39]. However, as usual, one considers the best approximation via the norm, so in this paper, we will discuss proximinality of subspaces of vector-valued MusielakOrlicz spaces via the norm. To proceed, we need to recall some definitions. Our results will be given in the next section.

○2014 Xu; licensee Springer. This is an Open Access article distributed under the terms of the Creative Commons Attribution License (http://creativecommons.org/licenses/by/2.0), which permits unrestricted use, distribution, and reproduction in any medium, provided the original work is properly cited. 
In what follows, $(A, \mathcal{A}, \mu)$ will be a $\sigma$-finite complete measure space. Suppose $D$ is a subset of $A$, let $\chi_{D}$ be the indicator function on $D$. Let $(X,\|\cdot\|)$ be a Banach space. The dual space of $X$ is the vector space $X^{*}$ of all continuous linear mappings from $X$ to $\mathbb{R}$ or $\mathbb{C}$. To avoid a double definition we let $\mathbb{K}$ be either $\mathbb{R}$ or $\mathbb{C}$.

Definition 1 A convex, left-continuous function $\varphi:[0, \infty) \rightarrow[0, \infty]$ with $\varphi(0)=0$, $\lim _{t \rightarrow 0^{+}} \varphi(t)=0, \lim _{t \rightarrow \infty} \varphi(t)=\infty$ is called a $\Phi$-function. It is called positive if $\varphi(t)>0$ for all $t>0$.

It is easy to see that if $\varphi$ is a $\Phi$-function, then it is nondecreasing on $[0, \infty)$.

Definition 2 Let $(A, \mathcal{A}, \mu)$ be a $\sigma$-finite complete measure space. A real function $\varphi: A \times$ $[0, \infty) \rightarrow[0, \infty]$ is called a generalized $\Phi$-function on $(A, \mathcal{A}, \mu)$ if

(a) $\varphi(y, \cdot)$ is a $\Phi$-function for all $y \in A$,

(b) $y \mapsto \varphi(y, t)$ is measurable for all $t \geq 0$.

If $\varphi$ is a generalized $\Phi$-function on $(A, \mathcal{A}, \mu)$, we write $\varphi \in \Phi(A, \mu)$.

Definition 3 Let $\varphi \in \Phi(A, \mu)$. Define

$$
\varrho_{\varphi}(f):=\int_{A} \varphi(y,\|f(y)\|) \mathrm{d} \mu(y)
$$

for strongly $\mu$-measurable functions $f: A \rightarrow X$. Then the Bochner-Musielak-Orlicz space $L^{\varphi}(A, X)$ is the collection of all strongly $\mu$-measurable functions $f: A \rightarrow X$ endowed with the norm:

$$
\|f\|_{L^{\varphi}(A, X)}:=\inf \left\{\lambda>0, \varrho_{\varphi}(f / \lambda) \leq 1\right\} .
$$

Let

$$
E^{\varphi}(A, X):=\left\{f \in L^{\varphi}(A, X): \rho_{\varphi}(\lambda f)<\infty \text { for all } \lambda>0\right\} .
$$

Definition 4 Let $\varphi \in \Phi(A, \mu)$. The function $\varphi$ is said to obey the $\Delta_{2}$-condition if there exists $K \geq 2$ such that

$$
\varphi(s, 2 t) \leq K \varphi(s, t)
$$

for all $s \in A$ and all $t \geq 0$.

When $X$ is $\mathbb{R}$ or $\mathbb{C}$, we simply denote $L^{\varphi}(A, X)$ by $L^{\varphi}(A)$, and $E^{\varphi}(A, X)$ by $E^{\varphi}(A)$. Usually, $E^{\varphi}(A, X)$ is a proper subspace of $L^{\varphi}(A, X)$. But when the $\varphi$ satisfies the $\Delta_{2}$-condition, they are the same. It is easy to see that $E^{\varphi}(A, X)=L^{\varphi}(A, X)$ is equivalent to $E^{\varphi}(A)=L^{\varphi}(A)$, this means that the equality depends only on $\varphi$.

We remark that $\rho_{\varphi}$ is a semimodular on the space of all $X$-valued strongly $\mu$-measurable functions on $A$. For a semimodular, we recommend the reader reference [6]. Let $\rho$ be a semimodular on vector space $E, E_{\rho}=\{x \in E: \rho(x / \lambda)<\infty$ for some $\lambda>0\},\|x\|_{\rho}=\inf \{\lambda>$ $0: \rho(x / \lambda) \leq 1\}$. We will use the following elementary result for a semimodular, which is Corollary 2.1.15 in [6]. 
Lemma 1 Let $\rho$ be a semimodular on $E, x \in E_{\rho}$.

(i) If $\|x\|_{\rho} \leq 1$, then $\rho(x) \leq\|x\|_{\rho}$.

(ii) If $1<\|x\|_{\rho}$, then $\|x\|_{\rho} \leq \rho(x)$.

Let $X$ be a Banach space and let $Y$ be a closed subspace of $X$. Then $Y$ is called proximinal in $X$ if for any $x \in X$ there exists $y \in Y$ such that

$$
\|x-y\|=\operatorname{dist}(x, Y)=\inf \{\|x-u\|: u \in Y\} .
$$

In this case $y$ is called a best approximation of $x$ in $Y$. If this best approximation is unique for any $x \in X$, then $Y$ is said to be Chebyshev.

For simplicity, we denote $\|\cdot\|_{L^{\varphi}(A, X)}$ or $\|\cdot\|_{L^{\varphi}(A)}$ by $\|\cdot\|_{\varphi}$. For $f \in L^{\varphi}(A, X), Y \subset X$, let

$$
\operatorname{dist}_{\varphi}\left(f, L^{\varphi}(A, Y)\right):=\inf \left\{\|f-g\|_{\varphi}: g \in L^{\varphi}(A, Y)\right\} .
$$

\section{Main results}

Firstly, we estimate $\operatorname{dist}_{\varphi}\left(f, L^{\varphi}(A, Y)\right)$.

Theorem 1 Let $Y$ be a subspace of Banach space X. Suppose $\varphi \in \Phi(A, \mu)$. For $f \in L^{\varphi}(A, X)$, define $\phi: A \rightarrow \mathbb{R}$ by $\phi(s):=\operatorname{dist}(f(s), Y)$. Then

(i) $\phi \in L^{\varphi}(A)$ and $\operatorname{dist}_{\varphi}\left(f, L^{\varphi}(A, Y)\right) \geq\|\phi\|_{\varphi}$;

(ii) $\operatorname{dist}_{\varphi}\left(f, L^{\varphi}(A, Y)\right)=\|\phi\|_{\varphi}$ for $f \in E^{\varphi}(A, X)$.

Proof (i) Given $f \in L^{\varphi}(A, X)$, we see that there exists a sequence of simple functions $\left\{f_{n}\right\}$ which converges to $f$ almost everywhere and in $L^{\varphi}(A, X)$. Since the distance function $d(x, Y)$ is a continuous function of $x \in X,\left\|f_{n}(s)-f(s)\right\| \rightarrow 0$ implies that $\mid \operatorname{dist}\left(f_{n}(s), Y\right)-$ $\operatorname{dist}(f(s), Y) \mid \rightarrow 0$. Moreover, each function $\phi_{n}: A \rightarrow \mathbb{R}$ defined by $\phi_{n}(s):=\operatorname{dist}\left(f_{n}(s), Y\right)$ is a simple function; therefore we conclude that $\phi$ is measurable. Now, for any $g \in L^{\varphi}(A, Y)$ and any $\lambda>0$,

$$
\begin{aligned}
\rho_{\varphi}(\lambda(f-g)) & =\int_{A} \varphi(s, \lambda\|f(s)-g(s)\|) \mathrm{d} \mu(s) \\
& \geq \int_{A} \varphi(s, \lambda \operatorname{dist}(f(s), Y)) \mathrm{d} \mu(s) \\
& =\rho_{\varphi}(\lambda \phi) .
\end{aligned}
$$

Thus, we have

$$
\|f-g\|_{\varphi} \geq\|\phi\|_{\varphi}
$$

This implies $\phi \in L^{\varphi}(A)$ and, by taking an infimum on $g \in L^{\varphi}(A, Y)$, we have

$$
\operatorname{dist}_{\varphi}\left(f, L^{\varphi}(A, Y)\right) \geq\|\phi\|_{\varphi} .
$$

(ii) We first assume that $f$ is a simple function. Let $f(s):=\sum_{i=1}^{m} \chi_{A_{i}} x_{i}$ where $\left\{A_{i}\right\}_{i=1}^{m}$ are disjoint measurable subsets in $A$ such that $0<\mu\left(A_{i}\right)<\infty$ and $0 \neq x_{i} \in X$ for $i \in\{1, \ldots, m\}$. Without loss of generality, we suppose that $\operatorname{dist}_{\varphi}\left(f, L^{\varphi}(A, Y)\right)=1$. Let $0<\epsilon<1$. Since $\phi(s) \leq$ 
$\|f(s)\|$, we have $\rho_{\varphi}(\lambda \phi) \leq \rho_{\varphi}(\lambda f)<\infty$ for any $\lambda>0$. Then, by the dominated convergence theorem, we find that there exists $\delta>0$ such that

$$
\int_{A_{i}} \varphi\left(s, \operatorname{dist}\left(x_{i}, Y\right)+\delta\right) \mathrm{d} \mu(s) \leq \int_{A_{i}} \varphi\left(s, \operatorname{dist}\left(x_{i}, Y\right)\right) \mathrm{d} \mu(s)+\frac{\epsilon}{m}, \quad \forall i \in\{1, \ldots, m\} .
$$

Now take $y_{i} \in Y$ such that $\left\|x_{i}-y_{i}\right\|<\operatorname{dist}\left(x_{i}, Y\right)+\delta$ for $i \in\{1, \ldots, m\}$. Let $g(s)=\sum_{i=1}^{m} \chi_{A_{i}} y_{i}$. Therefore $\|f-g\|_{\varphi} \geq \operatorname{dist}_{\varphi}\left(f, L^{\varphi}(A, Y)\right)=1$. By Lemma 1 , we see that

$$
\begin{aligned}
1 & \leq\|f-g\|_{\varphi} \leq \rho_{\varphi}(f-g)=\sum_{i=1}^{m} \int_{A_{i}} \varphi\left(s,\left\|x_{i}-y_{i}\right\|\right) \mathrm{d} \mu(s) \\
& \leq \sum_{i=1}^{m} \int_{A_{i}} \varphi\left(s, \operatorname{dist}\left(x_{i}, Y\right)+\delta\right) \mathrm{d} \mu(s) \\
& \leq \sum_{i=1}^{m}\left(\int_{A_{i}} \varphi\left(s, \operatorname{dist}\left(x_{i}, Y\right)\right) \mathrm{d} \mu(s)+\frac{\epsilon}{m}\right) \\
& =\int_{A} \varphi(s, \operatorname{dist}(f(s), Y)) \mathrm{d} \mu(s)+\epsilon .
\end{aligned}
$$

Thus, $\rho_{\varphi}(\phi) \geq 1-\epsilon$. Since $\epsilon$ is arbitrary, we have $\rho_{\varphi}(\phi) \geq 1$. By Lemma 1 again, we have $\|\phi\|_{\varphi} \geq 1$. This means that $\|\operatorname{dist}(f(\cdot), Y)\|_{\varphi} \geq \operatorname{dist}_{\varphi}\left(f, L^{\varphi}(A, Y)\right)$. Therefore, we have proved that $\|\operatorname{dist}(f(\cdot), Y)\|_{\varphi}=\operatorname{dist}_{\varphi}\left(f, L^{\varphi}(A, Y)\right)$ for simple functions.

Finally, let $f \in E^{\varphi}(A, X)$, there exists a sequence of simple functions $\left\{g_{n}\right\}_{n \in \mathbb{N}}$ convergent to $f \mu$-almost everywhere, $\left\|g_{n}(s)\right\| \leq\|f(s)\| \mu$-almost everywhere and $\left\|f-g_{n}\right\|_{\varphi} \rightarrow 0$ as $n$ tends to $\infty$. Let $\phi_{n}(s)=\operatorname{dist}\left(g_{n}(s), Y\right)$. From the previous proof, we have

$$
\left\|\phi_{n}\right\|_{\varphi}=\operatorname{dist}_{\varphi}\left(g_{n}, L^{\varphi}(A, Y)\right) .
$$

It is easy to see that $\operatorname{dist}_{\varphi}\left(g_{n}, L^{\varphi}(A, Y)\right) \rightarrow \operatorname{dist}_{\varphi}\left(f, L^{\varphi}(A, Y)\right)$ as $n \rightarrow \infty$. Since $\phi_{n}(s) \leq$ $\left\|g_{n}(s)\right\| \leq\|f(s)\| \mu$-almost everywhere, and $\phi_{n}(s) \rightarrow \phi(s) \mu$-almost everywhere as $n$ tends to $\infty$, by Lemma 2.3.16(c) in [6], we conclude that $\phi_{n} \rightarrow \phi$ in $L^{\varphi}(A)$. Hence, letting $n \rightarrow \infty$, we see that

$$
\|\phi\|_{\varphi}=\operatorname{dist}_{\varphi}\left(f, L^{\varphi}(A, Y)\right)
$$

which completes the proof of Theorem 1.

Corollary 1 Let $Y$ be a closed subspace of a Banach space X. Suppose $\varphi \in \Phi(A, \mu)$. An element $g$ of $L^{\varphi}(A, Y)$ is a best approximation to an element $f$ in $E^{\varphi}(A, X)$ if and only if $g(s)$ is a best approximation in $Y$ to $f(s)$ for almost every $s \in A$. Furthermore, if $\varphi$ satisfies the $\Delta_{2}$-condition, then an element $g$ of $L^{\varphi}(A, Y)$ is a best approximation to an element $f$ in $L^{\varphi}(A, X)$ if and only if $g(s)$ is a best approximation in $Y$ to $f(s)$ for almost every $s \in A$.

Corollary 2 Let $Y$ be a Chebyshev subspace of a Banach space X. Suppose $\varphi \in \Phi(A, \mu)$ satisfies the $\Delta_{2}$-condition. If $L^{\varphi}(A, Y)$ is proximinal in $L^{\varphi}(A, X)$, then it is a Chebyshev subspace of $L^{\varphi}(A, X)$.

Remark 1 Our results Theorem 1, Corollaries 1 and 2 cover the results for vector Orlicz spaces in [36]. Indeed, in [36] the authors only considered vector Orlicz spaces on finite 
measures. Analogous results for best simultaneous approximation were obtained in [37, 38] for vector Orlicz spaces on finite measures.

Next, we transfer the proximinality of $Y$ in $X$ to $L^{\varphi}(A, Y)$ in $L^{\varphi}(A, X)$. To do so, we need some preliminaries.

Lemma 2 Let $(A, \mathcal{A}, \mu)$ be a $\sigma$-finite complete measure space. Suppose $\varphi \in \Phi(A, \mu)$. Let $Y$ be a proximinal subspace of a Banach space X. Suppose $f \in L^{\varphi}(A, X)$ and $g$ is a strongly $\mu$-measurable function such that $g(s)$ is a best approximation to $f(s)$ from $Y$ for almost everywhere $s \in A$. Then $g$ is a best approximation to f from $L^{\varphi}(A, Y)$.

Proof Since $0 \in Y$, it follows that $\|g(s)\| \leq 2\|f(s)\| \mu$-almost everywhere. Thus, $g \in$ $L^{\varphi}(A, Y)$. For each $h \in L^{\varphi}(A, Y)$, by assumption we know that $\|f(s)-g(s)\| \leq\|f(s)-h(s)\|$ $\mu$-almost everywhere. So $\rho_{\varphi}(\lambda(f-g)) \leq \rho_{\varphi}(\lambda(f-h))$ for any $\lambda>0$. Thus, $\|f-g\|_{\varphi} \leq$ $\|f-h\|_{\varphi}$. This ends the proof.

Definition 5 Let $(T, \tau)$ be a Polish space (i.e. a topological space which is separable and completely metrizable). A set $Q \subset T$ is analytic if it is empty or if there exists a continuous mapping $f: \mathbb{N}^{\mathbb{N}} \rightarrow T$ satisfying $f\left(\mathbb{N}^{\mathbb{N}}\right)=Q$, where $\mathbb{N}^{\mathbb{N}}$ denotes the space of all infinite sequences of natural numbers endowed with the Tychonoff topology.

Definition 6 Let $(T, \tau)$ be a Polish space, $H$ a topological space and denote by $\sigma(\mathcal{A})$ the smallest $\sigma$-algebra containing all analytic subsets of $T$. Then a mapping $f: T \rightarrow H$ is said to be analytic measurable if $f^{-1}(C) \in \sigma(\mathcal{A})$ for every $C \in \mathcal{B}(H)$, where $\mathcal{B}(H)$ is for the Borel sets of $H$.

Definition 7 Let $H, T$ be topological spaces. Then a multifunction $F: H \rightarrow 2^{T}$ is said to be upper semi-continuous if for every $x \in H$ and for every open set $U$ satisfying $F(x) \subset U$, there exists an open neighborhood $V$ of $x$ such that $F(V) \subset U$.

Definition 8 A subset $C$ of a topological space $T$ is $\mathcal{K}$-analytic if it can be written as $C=\bigcup_{\sigma \in \mathbb{N}^{\mathbb{N}}} F(\sigma)$ for some upper semi-continuous mapping $F: \mathbb{N}^{\mathbb{N}} \rightarrow 2^{T}$ with compact values. In the case when $T$ is a Banach space endowed with its weak topology, $C$ is said to be weakly $\mathcal{K}$-analytic.

For the theory of $\mathcal{K}$-analytic sets, we recommend [40]. Specially, all reflexive and all separable Banach spaces are weakly $\mathcal{K}$-analytic. The following lemma is just Theorem 3.3 in [31].

Lemma 3 Let $(X,\|\cdot\|)$ be a real Banach space and let $Y$ be a proximinal, weakly $\mathcal{K}$-analytic convex subset of $X$. Then, for each closed and separable set $M \subset X$, there exists an analytic measurable mapping $h: M \rightarrow Y$ such that $h(M)$ is separable in $Y$ and $h(x)$ is a best approximation of $x$ in $Y$ for any $x \in M$.

Thus, following the argument of the proof of (i) $\rightarrow$ (ii) in [39, p.185], we have the following conclusion, the details being omitted. 
Theorem 2 Let $(A, \mathcal{A}, \mu)$ be a $\sigma$-finite complete measure space. Suppose $\varphi \in \Phi(A, \mu)$, and $Y$ is a weakly $\mathcal{K}$-analytic linear subspace of a real Banach space $X$. If $Y$ is proximinal in $X$, then $L^{\varphi}(A, Y)$ is proximinal in $L^{\varphi}(A, X)$.

Theorem 3 Let $(A, \mathcal{A}, \mu)$ be a $\sigma$-finite measure space. Suppose $\varphi \in \Phi(A, \mu)$ such that $E^{\varphi}(A)=L^{\varphi}(A)$. Let $Y$ be a linear subspace of a real Banach space $X$. If $L^{\varphi}(A, Y)$ is proximinal in $L^{\varphi}(A, X)$, then $Y$ is proximinal in $X$.

Proof Since the measure $\mu$ is $\sigma$-finite, let us choose positive measure set $Q$ such that $\chi_{Q} \in$ $L^{\varphi}(A)$. For any $x \in X$, let $f(t):=\chi_{Q}(t) \cdot x, t \in A$. Then $f \in L^{\varphi}(A, X)$. By the assumption, we know that there is a $g$ in $L^{\varphi}(A, Y)$ which is a best approximation element of $f$. Consequently, $g(s)$ is a best approximation to $f(s)$ in $Y$ for almost every $s \in A$ by Corollary 1 . Therefore, there is a best approximation element of $x$ in $Y$. Thus, $Y$ is proximinal in $X$.

From Theorems 2 and 3, we deduce the following corollary.

Corollary 3 Let $(A, \mathcal{A}, \mu)$ be a $\sigma$-finite complete measure space. Suppose $\varphi \in \Phi(A, \mu)$ such that $E^{\varphi}(A)=L^{\varphi}(A)$. Let $Y$ be a weakly $\mathcal{K}$-analytic linear subspace of a real Banach space $X$. Then the following conditions are equivalent:

(i) $Y$ is proximinal in $X$;

(ii) $L^{\varphi}(A, Y)$ is proximinal in $L^{\varphi}(A, X)$.

Remark 2 An analog to Corollary 3 in terms of a modular was obtained in [39].

Finally, we give a characterization of proximinity of $L^{\varphi}(A, Y)$ in $L^{\varphi}(A, X)$ via the proximinity of $L^{1}(A, Y)$ in $L^{1}(A, X)$. When $L^{\varphi}(A, X)$ is a Bochner-Lebesgue space, which was obtained in [29] and [32,33] on finite measure spaces and $\sigma$ finite measure spaces, respectively. $L^{\varphi}(A, X)$ is a Bochner-Orlicz space, which was discussed in [35].

Theorem 4 Let $(A, \mathcal{A}, \mu)$ be a $\sigma$-finite complete measure space. Suppose $\varphi \in \Phi(A, \mu)$ such that the set of simple functions, $S(A, \mu)$, satisfies $S(A, \mu) \subset L^{\varphi^{*}}(A, \mu)$, where $\varphi^{*}$ is the conjugate function of $\varphi$ (see [6]). Let $Y$ be a closed subspace of a Banach space $X$. If $L^{1}(A, Y)$ is proximinal in $L^{1}(A, X)$, then $L^{\varphi}(A, Y)$ is proximinal in $L^{\varphi}(A, X)$.

Proof Since $A$ is $\sigma$-finite, we may write $A=\bigcup_{i=1}^{\infty} A_{i}$, where $\left\{A_{i}\right\}$ is a sequence of disjoint measurable sets each of finite measure. Let $f \in L^{\varphi}(A, X)$. For any $n \in \mathbb{N}$, since $\mu\left(A_{n}\right)<\infty$, then $\chi_{A_{n}} \in L^{\varphi^{*}}(A)$. Thus, by the norm conjugate formula (see Corollary 2.7.5 in [6]), we find that $f \chi_{A_{n}} \in L^{1}(A, X)$. By assumption, we know that there exists $g_{n} \in L^{1}(A, Y)$ such that

$$
\left\|f \chi_{A_{n}}-g_{n}\right\|_{L^{1}} \leq\left\|f \chi_{A_{n}}-h\right\|_{L^{1}}, \quad \forall h \in L^{1}(A, Y) .
$$

By Corollary 1, we have, for all $y \in Y$,

$$
\left\|f(t) \chi_{A_{n}}-g_{n}(t)\right\| \leq\left\|f(t) \chi_{A_{n}}-y\right\|
$$

$\mu$-almost everywhere. Therefore $g_{n}(t)=0 \mu$-almost every $t \in A_{n}^{c}$. Let $g=\sum_{n=1}^{\infty} g_{n}$. Since $f=\sum_{n=1}^{\infty} f \chi_{A_{n}}$, it follows that for all $h \in L^{\varphi}(A, Y)$,

$$
\|f(t)-g(t)\| \leq\|f(t)-h(t)\|
$$


$\mu$-almost everywhere. Because $0 \in Y$, it follows that $\|g(t)\| \leq 2\|f(t)\|$. Thus, $g \in L^{\varphi}(A, Y)$ and

$$
\|f-g\|_{\varphi} \leq\|f-h\|_{\varphi}
$$

for all $h \in L^{\varphi}(A, Y)$. This finishes the proof.

Theorem 5 Let $(A, \mathcal{A}, \mu)$ be a $\sigma$-finite complete measure space. Suppose $\varphi \in \Phi(A, \mu)$ satisfies $E^{\varphi}(A)=L^{\varphi}(A)$ and, for each $t \in A, \varphi(t, \cdot)$ is strictly increasing. Let $Y$ be a closed subspace of a Banach space $X$. If $L^{\varphi}(A, Y)$ is proximinal in $L^{\varphi}(A, X)$, then $L^{1}(A, Y)$ is proximinal in $L^{1}(A, X)$.

Proof We use the idea from [35]. Indeed in [35] the authors only considered Banach space valued Orlicz spaces on the unit interval. Since, for each $t \in A, \varphi(t, \cdot)$ is strictly increasing, let $\varphi^{-1}(t, \cdot)$ be its inverse function, which means, for each $s \in[0, \infty), \varphi\left(t, \varphi^{-1}(t, s)\right)=s$. Define the map $J: L^{1}(A, X) \rightarrow L^{\varphi}(A, X)$ by setting

$$
J(f)(t):= \begin{cases}\frac{\varphi^{-1}(t,\|f(t)\|)}{\|f(t)\|} f(t), & f(t) \neq 0 ; \\ 0, & f(t)=0 .\end{cases}
$$

Then $\|J(f)(t)\|=\varphi^{-1}(t,\|f(t)\|)$. Therefore $\rho_{\varphi}(J(f))=\|f\|_{L^{1}}$. So $J$ is injective. Moreover, if $g \in L^{\varphi}(A, X)$, let

$$
f(t):= \begin{cases}\frac{\varphi(t,\|g(t)\|) \mid}{\|g(t)\|} g(t), & g(t) \neq 0 \\ 0, & g(t)=0 .\end{cases}
$$

Then $f(t) \in X$ and $\|f(t)\|=\varphi(t,\|g(t)\|)$. Thus, $f \in L^{1}(A, X)$. In addition, for $g(t) \neq 0$,

$$
J(f)(t)=\frac{\varphi^{-1}(t, \varphi(t,\|g(t)\|))}{\varphi(t,\|g(t)\|)} f(t)=\frac{\|g(t)\|}{\varphi(t,\|g(t)\|)} f(t)=g(t) .
$$

If $g(t)=0$, then $f(t)=0$ also, thus $J(f)(t)=0=g(t)$. Hence $J$ is surjective and $J\left(L^{1}(A, Y)\right)=$ $L^{\varphi}(A, Y)$ also.

Now, let $f \in L^{1}(A, X)$. Without loss of generality we may suppose that $f(t) \neq 0 \mu$-almost everywhere, for otherwise we can restrict our measure to the support of $f$. Since $J(f) \in$ $L^{\varphi}(A, X)$, by the assumption, we know that there exists some $g \in L^{1}(A, Y)$ such that

$$
\|J(f)-J(g)\|_{\varphi} \leq\|J(f)-J(v)\|_{\varphi}
$$

for all $v \in L^{1}(A, Y)$. By Corollary 1 , we see that, for all $y \in Y$,

$$
\|J(f)(t)-J(g)(t)\| \leq\|J(f)(t)-y\|
$$

$\mu$-almost everywhere. Multiplying both sides of the last inequality by $\frac{\|f(t)\|}{\varphi^{-1}(t,\|f(t)\|)}$, we obtain, for all $y \in Y$,

$$
\left\|f(t)-\frac{\|f(t)\|}{\varphi^{-1}(t,\|f(t)\|)} \frac{\varphi^{-1}(t,\|g(t)\|)}{\|g(t)\|} g(t)\right\| \leq\|f(t)-y\| .
$$


Let $h(t)=\frac{\|f(t)\|}{\varphi^{-1}(t,\|f(t)\|)} \frac{\varphi^{-1}(t,\|g(t)\|)}{\|g(t)\|} g(t)$. Since $h(t)$ is a best approximation of $f(t)$ in $Y$, and $0 \in Y$, it follows that $\|h(t)\| \leq 2\|f(t)\|$. Therefore, $h \in L^{1}(A, Y)$. Thus, for all $w \in L^{1}(A, Y)$,

$$
\|f(t)-h(t)\| \leq\|f(t)-w(t)\|
$$

$\mu$-almost everywhere. Thus, by Corollary $1 h$ is a best approximation of $f$ in $L^{1}(A, Y)$. This finishes the proof.

From Theorems 4 and 5, we deduce the following corollary.

Corollary 4 Let $(A, \mathcal{A}, \mu)$ be a $\sigma$-finite complete measure space. Suppose $\varphi \in \Phi(A, \mu)$ such that $E^{\varphi}(A)=L^{\varphi}(A)$, for each $t \in A, \varphi(t, \cdot)$ is strictly increasing and the set of simple functions $S(A, \mu)$ satisfies $S(A, \mu) \subset L^{\varphi^{*}}(A, \mu)$. Let $Y$ be a closed subspace of a Banach space $X$. Then the following conditions are equivalent:

(i) $L^{1}(A, Y)$ is proximinal in $L^{1}(A, X)$;

(ii) $L^{\varphi}(A, Y)$ is proximinal in $L^{\varphi}(A, X)$.

Remark 3 When $(A, \mu)$ is a finite measure and $\varphi$ is a Orlicz function that satisfies the $\Delta_{2}$ condition, the result of Corollary 4 was obtained in [36]. While $(A, \mu)$ is the unit interval and $\varphi$ is a Young function that satisfies the $\Delta_{2}$-condition, the result of Corollary 4 was obtained in [35].

\section{Competing interests}

The author declare that he has no competing interests.

\section{Acknowledgements}

The author would like to thank the referee for carefully reading which made the presentation more readable and for his or her suggestion for references [36-38]. The author was supported by the National Natural Science Foundation of China (Grant No. 11361020) and the National Natural Science Foundation of Hainan Providence (113004).

Received: 22 December 2013 Accepted: 27 March 2014 Published: 09 Apr 2014

\section{References}

1. Musielak, J: Orlicz Spaces and Modular Spaces. Springer, Berlin (1983)

2. Kováčik, O, Rákosník, J: On spaces $L^{p(x)}$ and $W^{k, p(x)}$. Czechoslov. Math. J. 41, 592-618 (1991)

3. Almeida, A, Drihem, D: Maximal, potential and singular type operators on Herz spaces with variable exponents. J. Math. Anal. Appl. 394, 781-795 (2012)

4. Almeida, A, Hästö, P: Besov spaces with variable smoothness and integrability. J. Funct. Anal. 258, 1628-1655 (2010)

5. Diening, L, Hästö, P, Roudenko, S: Function spaces of variable smoothness and integrability. J. Funct. Anal. 256, 1731-1768 (2009)

6. Diening, L, Harjulehto, P, Hästö, P, Růžička, M: Lebesgue and Sobolev Spaces with Variable Exponents. Springer, Berlin (2011)

7. Harjulehto, P, Hästö, P, Le, UV, Nuortio, M: Overview of differential equations with non-standard growth. Nonlinear Anal. 72, 4551-4574 (2010)

8. Izuki, M: Boundedness of sublinear operators on Herz spaces with variable exponent and application to wavelet characterization. Anal. Math. 36, 33-50 (2010)

9. Izuki, M: Boundedness of commutators on Herz spaces with variable exponent. Rend. Circ. Mat. Palermo 59, 199-213 (2010)

10. Izuki, M: Vector-valued inequalities on Herz spaces and characterizations of Herz-Sobolev spaces with variable exponent. Glas. Mat. 45, 475-503 (2010)

11. Kempka, H: 2-Microlocal Besov and Triebel-Lizorkin spaces of variable integrability. Rev. Mat. Complut. 22, 227-251 (2009)

12. Kempka, H: Atomic, molecular and wavelet decomposition of generalized 2-microlocal Besov spaces. J. Funct. Spaces Appl. 8, 129-165 (2010)

13. Nakai, E, Sawano, Y: Hardy spaces with variable exponents and generalized Campanato spaces. J. Funct. Anal. 262 3665-3748 (2012)

14. Samko, S: Variable exponent Herz spaces. Mediterr. J. Math. 10, 2007-2025 (2013)

15. Xu, J: Variable Besov and Triebel-Lizorkin spaces. Ann. Acad. Sci. Fenn., Math. 33, 511-522 (2008) 
16. $\mathrm{Xu}, \mathrm{J}$ : The relation between variable Bessel potential spaces and Triebel-Lizorkin spaces. Integral Transforms Spec. Funct. 19, 599-605 (2008)

17. Cheng, C, Xu, J: Geometric properties of Banach space valued Bochner-Lebesgue spaces with variable exponent. J. Math. Inequal. 7, 461-475 (2013)

18. Cao, J, Chang, DC, Yang, D, Yang, S: Weighted local Orlicz-Hardy spaces on domains and their applications in inhomogeneous Dirichlet and Neumann problems. Trans. Am. Math. Soc. 365, 4729-4809 (2013)

19. Liang, Y, Huang, J, Yang, D: New real-variable characterizations of Musielak-Orlicz Hardy spaces. J. Math. Anal. Appl. 395, 413-428 (2012)

20. Yang, D, Yang, S: Orlicz-Hardy spaces associated with divergence operators on unbounded strongly Lipschitz domains of $\mathbb{R}^{n}$. Indiana Univ. Math. J. 61, 81-129(2012)

21. Yang, D, Yang, S: Local Hardy spaces of Musielak-Orlicz type and their applications. Sci. China Math. 55, 1677-1720 (2012)

22. Yang, D, Yang, S: Musielak-Orlicz-Hardy spaces associated with operators and their applications. J. Geom. Anal. 24 495-570 (2014)

23. Chen, Y, Levine, S, Rao, R: Variable exponent, linear growth functionals in image restoration. SIAM J. Appl. Math. 66, 1383-1406 (2006)

24. Li, F, Li, Z, Pi, L: Variable exponent functionals in image restoration. Appl. Math. Comput. 216, 870-882 (2010)

25. Růžička, M: Electrorheological Fluids: Modeling and Mathematical Theory. Springer, Berlin (2000)

26. Abu-Sirhan, E: Simultaneous approximation in function spaces. In: Neamtu, M, Schumaker, L (eds.) Approximation Theory XIII: San Antonio, pp. 321-329 (2010)

27. Abu-Sirhan, E: Best simultaneous approximation in function and operator spaces. Turk. J. Math. 36, 101-112 (2012)

28. Khalil, R: Best approximation in $L_{p}(I, X)$. Math. Proc. Camb. Philos. Soc. 94, 277-279 (1983)

29. Khalil, R, Deeb, W: Best approximation in $L_{p}(I, X)$. J. Approx. Theory 59, 296-299 (1989)

30. Khalil, R, Saidi, F: Best approximation in $L_{1}(I, X)$. Proc. Am. Math. Soc. 123, 183-190 (1995)

31. Light, WA: Proximinality in $L_{p}(S, Y)$. Rocky Mt. J. Math. 19, 251-259 (1989)

32. Mendoza, J: Proximinality in $L_{p}(\mu, X)$. J. Approx. Theory 93, 331-343 (1998)

33. You, $Z Y, G$,,$T X$ : Pointwise best approximation in the space of strongly measurable functions with applications to best approximation in $L^{P}(\mu, X)$. J. Approx. Theory 78, 314-320 (1994)

34. Xu, J: Proximinality in Banach space valued Bochner-Lebesgue spaces with variable exponent (submitted)

35. Khandaqji, M, Khalil, R, Hussein, D: Proximinality in Orlicz-Bochner function spaces. Tamkang J. Math. 34, 71-75 (2003)

36. Al-Minawi, H, Ayesh, S: Best approximation in Orlicz spaces. Int. J. Math. Math. Sci. 14, $245-252$ (1991)

37. Khandaqji, M, Al-Sharif, S: Best simultaneous approximation in Orlicz spaces. Int. J. Math. Math. Sci. 2007, Article ID 68017 (2007)

38. Shen, ZS, Yang, ZY: Best simultaneous approximation in $L^{\Phi}(I, X)$. J. Math. Res. Expo. 30, 863-868 (2010)

39. Micherda, B: On proximinal subspaces of vector-valued Orlicz-Musielak spaces. J. Approx. Theory 174, $182-191$ (2013)

40. Kakol, J, Kubiś, W, López-Pellicer, M: Descriptive Topology in Selected Topics of Functional Analysis. Springer, Berlin (2011)

10.1186/1029-242X-2014-146

Cite this article as: Xu: Proximinality in Banach space valued Musielak-Orlicz spaces. Journal of Inequalities and Applications 2014, 2014:146

\section{Submit your manuscript to a SpringerOpen ${ }^{\circ}$ journal and benefit from:}

- Convenient online submission

- Rigorous peer review

- Immediate publication on acceptance

- Open access: articles freely available online

- High visibility within the field

- Retaining the copyright to your article 